\title{
Gegenwind für die Erneuerbaren - Räumliche Neuorientierung der Wind-, Solar- und Bioenergie vor dem Hintergrund einer verringerten Akzeptanz sowie zunehmender Flächennutzungskonflikte im ländlichen Raum
}

\author{
Stephan Bosch · Gerd Peyke
}

Eingegangen: 30. Juli 2010 / Angenommen: 7. Februar 2011 / Online publiziert: 22. März 2011

(C) Springer-Verlag 2011

\begin{abstract}
Zusammenfassung Der starke Ausbau von erneuerbaren Energien hat innerhalb nur weniger Jahre eine Kulturlandschaft neuen Typs hervorgebracht. Gewohnte Landschaftsbilder, die vor allem von parzellierten landwirtschaftlichen Nutzflächen, inselartigen Waldgebieten und räumlich konzentrierten Siedlungsflächen geprägt waren, werden in zunehmendem Maße durch dezentrale Kraftwerke wie Windparks, Photovoltaik-Freiflächenanlagen und Biomassekraftwerke ergänzt. Von einer Entwicklung hin zu reinen Energielandschaften, wie es seitens Heimatpflegevereinen und Naturschutzverbänden befürchtet wird, ist man jedoch weit entfernt. Eine gegenüber den erneuerbaren Energien restriktive Regionalplanung, jährliche Korrekturen an der Vergütungsstruktur des Erneuerbare-Energien-Gesetzes sowie eine degressiv ausgerichtete Förderung setzen einem regenerativen Energiesystem deutliche räumliche Grenzen. Dennoch hat der bisherige Ausbau den Nutzungsdruck auf den ländlichen Raum stark erhöht und zu neuen Dimensionen der Flächennutzungskonkurrenz geführt. Diskussionen um die zukünftige Bedeutung des ländlichen Raumes als Standort für neue Technologien erscheinen daher angebracht.
\end{abstract}

Schlüsselwörter Ressource Raum ·

Flächennutzungskonflikte · Dezentrale Energieerzeugung · Akzeptanz $\cdot$ Regionalplanung $\cdot$ Kulturlandschaft .

Ländlicher Raum

S. Bosch $(\varangle) \cdot$ Prof. Dr. G. Peyke

Lehrstuhl für Humangeographie und Geoinformatik, Universität

Augsburg, Universitätsstraße 10, 86159 Augsburg, Deutschland

E-Mail: stephan.bosch@geo.uni-augsburg.de

Prof. Dr. G. Peyke

E-Mail: gerd.peyke@geo.uni-augsburg.de
Blowback for Renewables - Spatial Reorientation of Wind-, Solar- and Bioenergy Against the Backdrop of Declining Acceptance and Increasing Conflicts of Usage of Acreage in the Rural Zone

\begin{abstract}
The forced expansion of renewable energy sources has induced within a few years time a cultural landscape of a new kind. The traditional natural scenery, dominated by agricultural parcels, islands of forest, and condensed areas of settlement, is continually being amended by decentralized power plants such as wind parks, solar and biomass power plants. Still we are far from the development of pure energy landscapes, as it is feared by local historic associations and nature conservation organisations. Restrictive regional planning, annual amendments to the principles of remuneration given in the Renewable Energies Act, as well as declining government aid are putting hard spatial strain on the renewable energy system. Yet pressure on the usage of rural space has been augmented by the present development and has led to new dimensions of competition for the usage of acreage. Thus, a discussion of the future role of rural areas as a location for new technologies seems appropriate.
\end{abstract}

Keywords Spatial resources - Conflicts of usage of acreage $\cdot$ Decentralized energy production - Acceptance Regional planning $\cdot$ Cultural landscape $\cdot$ Rural zone

\section{Stellenwert der erneuerbaren Energien}

Vor dem Hintergrund klimawirksamer Emissionen von Kohlekraftwerken, schwindender konventioneller Erdölreserven und der Konzentration von Lagerstätten in wenigen, politisch instabilen Regionen (Rempel 2008: 23 ff.) 
erscheint der Aufbau eines Fördersystems für erneuerbare Energien als eine logische Konsequenz. Elf Jahre nach der Einführung des Erneuerbare-Energien-Gesetzes (EEG) haben regenerative Technologien zwar einen starken, in der Kulturlandschaft deutlich sichtbaren Ausbau erfahren, die große Bedeutung von Erdöl, Kohle, Erdgas und Uran ist dennoch ungebrochen. Derzeit haben fossile und nukleare Energieträger noch einen Anteil von über $90 \%$ am Primärenergieverbrauch. Betrachtet man die Zusammensetzung des Anteils von erneuerbaren Energien am Primärenergieverbrauch, so wird die herausragende Stellung der Bioenergie sichtbar (vgl. Abb. 1). Im Strombereich jedoch liegt die Windenergie mit etwa $44 \%$ deutlich vor der Bioenergie mit $29 \%$ und der Wasserkraft mit $23 \%$ (vgl. Abb. 2). Die Beiträge von Solarenergie und Geothermie sind marginal.

In Deutschland wird der Ausbau von erneuerbaren Energien in erster Linie über das EEG und das ErneuerbareEnergien-Wärmegesetz (EEWärmeG) gesteuert. Einen weiteren Baustein stellt das am 28. September 2010 veröffentlichte Energiekonzept der Bundesregierung - im Übrigen das erste Energiekonzept seit der Ölkrise 1973 - dar. Die Finanzierung dieses Konzeptes erfolgt durch den Emissionshandel sowie die Kernbrennstoffsteuer. $\mathrm{Zu}$ betonen ist, dass es sich um ein umfassendes Energiekonzept für den Strom-, Wärme- sowie Verkehrssektor und keineswegs nur um ein ,Laufzeitverlängerungskonzept ${ }^{`}$ handelt. Im Mittelpunkt des Konzepts stehen vor allem die Klimaschutzziele. Die erneuerbaren Energien werden als tragende
Säule bezeichnet und ihr kosteneffizienter Ausbau über die Weiterentwicklung des EEG angestrebt. Mittels Kreditprogrammen der Kreditanstalt für Wiederaufbau (KfW) sowie beschleunigten Planungs- und Genehmigungsverfahren steht dabei vor allem die Offshore-Windenergie im Fokus. Zur Integration des steigenden Anteils an Regenerativstrom ist es zudem notwendig, den Netzausbau voranzutreiben. Eine Schlüsselstellung kommt der Energieeffizienz zu, denn bis zum Jahr 2050 soll der Energieverbrauch Deutschlands halbiert werden. Der Markt für Effizienzdienstleistungen muss hierzu ausgebaut werden. Ein weiteres Handlungsfeld stellt die Sanierung des Gebäudebestandes dar, nicht zuletzt entfallen $40 \%$ der benötigten Energie auf Gebäude. Den kontrovers diskutierten Teil des Energiekonzeptes bilden die Beschlüsse zur Verlängerung der Laufzeiten von Kernkraftwerken. Vor 1980 errichtete Kraftwerksblöcke werden um acht Jahre, später ans Netz angeschlossene um 14 Jahre länger laufen (Schultz 2010: 10 ff.).

Die Europäische Union vertritt die Ansicht, dass die Ziele beim Ausbau von erneuerbaren Energien - d. h. ein Anteil von erneuerbaren Quellen am Endenergieverbrauch von insgesamt $20 \%$ sowie ein für jeden Mitgliedstaat verbindlicher Biokraftstoff-Mindestanteil im Verkehrssektor von $10 \%$ bis zum Jahr 2020 - nur auf Gemeinschaftsebene zu erreichen sind. Grundlage des Energiekonzeptes der Bundesregierung bildet daher eine von den Mitgliedstaaten umzusetzende Richtlinie. Neben klaren Vorgaben besteht dabei noch ausreichend Freiraum für spezifische, einzel-
Abb. 1 Zusammensetzung des Anteils von erneuerbaren Energien am Primärenergieverbrauch in Deutschland. (Quelle: Eigene Darstellung nach Arbeitsgemeinschaft Energiebilanzen 2009: 1)

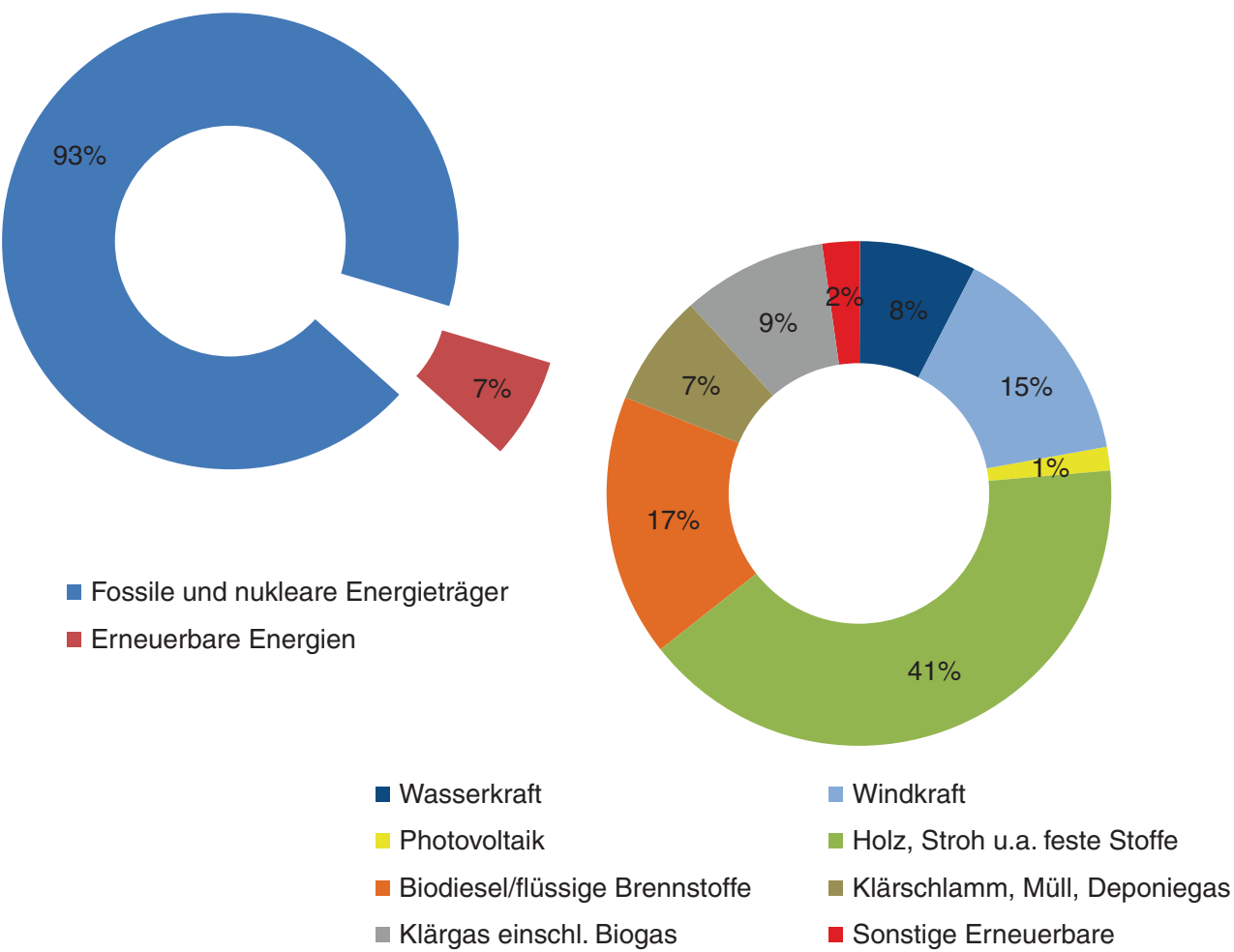


Abb. 2 Zusammensetzung des Anteils von erneuerbaren Energien an der Stromproduktion in Deutschland. (Quelle: Eigene Darstellung nach Arbeitsgemeinschaft Energiebilanzen 2009: 1)
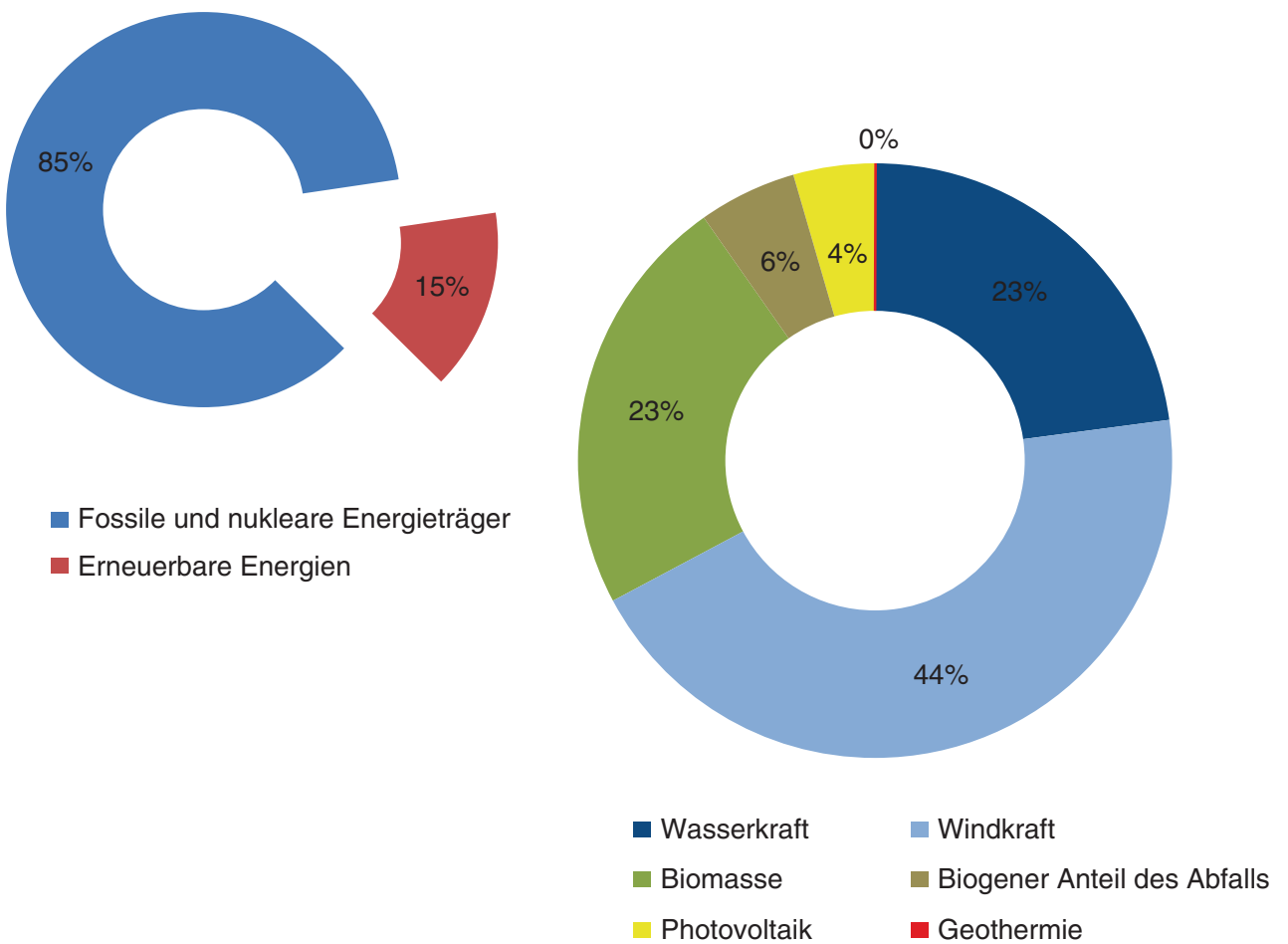

staatliche Konzeptionen. Von entscheidender Bedeutung ist die EU-Richtlinie 2009/28/EG. Ihr Sinn besteht darin, Maßnahmen für Strom, Wärme, Kälte und Kraftstoffe mittels einer Richtlinie zu bündeln. Speziell die Integration des Wärme- und Kältesektors ist aus Sicht der EU von immenser Bedeutung, denn die Vergangenheit habe gezeigt, dass Fortschritte beim Ausbau nur erzielt werden konnten, wenn die EU zuvor Rechtsvorschriften geschaffen hatte. In der Tat führten die EU-Richtlinien 2001/77/EG und 2003/30/ EG, die lediglich Vorgaben für den Strom- bzw. Kraftstoffsektor enthielten, zu einem Stillstand beim Ausbau von erneuerbaren Energien im Wärme- und Kältebereich. Grundsätzlich offenbart die EU-Richtlinie 2009/28/EG, dass die Ziele für den Ausbau von erneuerbaren Energien in unmittelbarem Zusammenhang mit klimapolitischen Erfordernissen stehen. Es wird betont, dass die Energiepolitik ein entscheidendes Instrument zur Umsetzung von Strategien hinsichtlich der Abmilderung des Klimawandels darstellt (Europäische Union 2009: 16 ff.).

Trotz der zahlreichen Initiativen auf nationaler und europäischer Ebene verläuft der Ausbau von erneuerbaren Energien in Deutschland nicht zufriedenstellend. Die gegenwärtige Tendenz zu einer verringerten Akzeptanz gegenüber einem weiteren Ausbau sowie die Zunahme von Konflikten widerstrebender Flächennutzungsinteressen sorgt in der Energiebranche zusehends für Pessimismus und verlangt angesichts der steigenden Energieabhängigkeit Deutschlands nach einer räumlichen Steuerung, die der großen politischen Bedeutung von erneuerbaren Energien Rechnung trägt. Zwar geben die speziell für Großunternehmen lukrativen Standortalternativen im Offshore-Bereich bzw. in Wüstengebieten Anlass zu der Vermutung, dass die Hoffnung auf die Etablierung eines nachhaltigen Energiesystems nicht gänzlich unbegründet ist. Zur Bewahrung des dezentralen Charakters eines regenerativen Energiesystems ist es jedoch notwendig, die Bedeutung des ländlichen Raumes als Standort für erneuerbare Energien zu stärken. Nur dort wird es mittelständischen Unternehmen aufgrund der relativ geringen Investitionskosten langfristig möglich sein, im Wettbewerb um energetisch hochwertige Flächen bestehen zu können. Die Möglichkeit des Zugriffs auf diese Flächen wird dabei entscheidend von der Ausgestaltung raumordnungs-, planungs- und zulassungsrechtlicher Instrumentarien abhängen.

Ziel des Aufsatzes ist es, die entscheidenden raumordnungsrechtlichen und die durch den Faktor Akzeptanz gesteuerten Ursachen für die räumlichen Einschränkungen von erneuerbaren Energien im ländlichen Raum zu verdeutlichen. Darüber hinaus werden Standortstrategien der Wind-, Solar- und Bioenergiebranche beschrieben, die als Reaktion auf Flächenengpässe im Binnenland zu verstehen sind und möglicherweise in einer Abschwächung des dezentralen Charakters des regenerativen Energiesystems münden. Schließlich werden Lösungsstrategien aufgezeigt, die eine nachhaltige Energieproduktion auch im ländlichen Raum in verstärktem Maße ermöglichen. 


\section{Räumliche Engpässe beim Ausbau der Windenergie}

\subsection{Relevanz länderspezifischer Regelungen}

Aus raumplanerischer Perspektive kommt der Windenergie eine Sonderstellung unter den erneuerbaren Energien zu. Einerseits erfahren Windkraftanlagen eine baurechtliche Privilegierung im Außenbereich von Gemeinden, andererseits existieren gebietsbezogene Nutzungsregelungen in Form von Vorranggebieten für die Windenergie. In einem dicht besiedelten Land wie Deutschland sind dem Ausbau von Windkraftanlagen seitens der Raumplanung dennoch klare Grenzen gesetzt. Die Flächenpotenziale werden durch gemeinschaftsrechtliche (z. B. NATURA 2000), fachrechtliche (z. B. Wasserschutzgebiete, Naturdenkmale) und raumordnungsrechtliche Festlegungen (z. B. Rohstoffsicherung, Erholung) sowie durch den vorgegebenen Abstand zu Siedlungskörpern eingeschränkt (vgl. Brücher 2008: 8). Dennoch geht die Agentur für Erneuerbare Energien (2009: 9) davon aus, dass die Stromproduktion aus der Onshore-Windenergie bis 2020 auf 112,1 Mrd. kWh pro Jahr gesteigert werden kann. Berechnet man die Abstandsflächen bei Windkraftanlagen von 6 bis 7 ha pro Megawatt mit ein, so würde sich der Flächenanspruch der Windenergie um etwa 100.000 ha auf dann insgesamt 270.000 ha erhöhen (vgl. Abb. 3). In Abhängigkeit von länderspezifischen Regelungen bezüglich der zulässigen Gesamthöhe von Windkraftanlagen kann dieser Wert jedoch variieren. Durch Re-Powering, d. h. den Ersatz leistungsschwacher durch eine geringere Anzahl leistungsstarker Anlagen mit größeren Nabenhöhen können die Flächenerträge erheblich gesteigert werden (Bundesverband WindEnergie 2010: 1 f.). Allein durch Aufstockung einer $100 \mathrm{~m}$ hohen Anlage der 2-MW-Klasse (MW=Megawatt) um 70 m wird im Durchschnitt ein zusätzlicher Ertrag von 2,1 Mio. kWh pro Jahr generiert (vgl. Abb. 4).

Die Prognosen der Agentur für Erneuerbare Energien können als optimistisch angesehen werden, wenn man bedenkt, dass die Akzeptanz eines weiteren Ausbaus von Windkraftanlagen regional teils erheblich schwindet. In Brandenburg gibt es beispielsweise eine Volksinitiative, deren Ziel es ist, den Abstand von Windkraftanlagen zu Siedlungen von 1000 auf $1500 \mathrm{~m}$ zu erhöhen. Regionalversammlungen greifen die Bedenken in der Bevölkerung auf und verhalten sich bei der Ausweisung von Flächen äußerst restriktiv (Weinhold 2009: 18). Windkraftbefürworter kritisieren diese Haltung und betonen, dass die Umsetzung der Energiestrategie 2020 lediglich 1,9\% $\left(555 \mathrm{~km}^{2}\right)$ der Landesfläche Brandenburgs in Anspruch nehmen würde und eine Änderung des Landeswaldgesetzes die Möglichkeit biete, von Nutzungsinteressen weniger beanspruchte Kiefernforste in Standortentscheidungsprozesse mit einbeziehen zu können (Landesregierung Brandenburg 2009: 2 ff.). In RheinlandPfalz beschäftigt man sich bereits seit längerem mit dem Gedanken, der Windenergie Waldstandorte in größerem Ausmaße zu eröffnen. Die Entwicklung von Windkraftanlagen mit immer größeren Nabenhöhen kommt dieser Strategie zu Gute, denn dadurch ist es möglich, die Turbinen in Höhen, deren Luftströmungen nur noch in geringem Maße der Rauigkeit eines Waldgebietes ausgesetzt sind, zu platzieren (Tobias/Jung/Fallen et al. 2003: 3 ff.). Eine Entschärfung des Konflikts in Brandenburg könnte auch dadurch erreicht werden, indem man die raumordnungsrechtliche Vorgabe, dass Eignungsgebiete für Windenergie mindestens fünf Kilometer voneinander entfernt sein müssen, korrigiert. Folglich könnte sich der Ausbau auf die Flächen mit den höchsten Windgeschwindigkeiten konzentrieren. Die
Abb. 3 Potenzieller Anteil von erneuerbaren Energien am Endenergieverbrauch bis 2020 und Flächenbelegung. (Quelle: Eigene Darstellung nach Agentur für Erneuerbare Energien 2009: $6 \mathrm{f}$.)

\begin{tabular}{|l|l|l|}
\hline Energieträger & Potenzial 2020 & Flächenverbrauch \\
\hline Windenergie & Strom: $18,8 \%$ & 270.000 ha \\
\hline Solarenergie & Strom: $6,6 \%$ & Freifläche: 10.500 ha \\
\hline Geothermie & Wärme: $2,6 \%$ & Gebäude : 37.000 ha \\
\hline Bioenergie & Strom: $0,6 \%$ & 960.000 ha [unterirdisch] \\
\hline & Wärme: $3,6 \%$ & \\
\hline Strom: $9,1 \%$ & 3,7 Mio. ha \\
\hline Wasserkraft & Wärme: $13,1 \%$ & \\
\hline & Kraftstoff: $21,4 \%$ & \\
\hline & Strom: $5,4 \%$ & \\
\hline
\end{tabular}


Abb. 4 Zusammenhang zwischen Höhe von Windkraftanlagen und Leistung. (Quelle: Eigene Darstellung nach Bundesverband WindEnergie 2010: $1 \mathrm{f}$.)

\begin{tabular}{|l|l|l|l|}
\hline Gesamthöhe & $100 \mathrm{~m}$ & $140 \mathrm{~m}$ & $170 \mathrm{~m}$ \\
\hline Nennleistung & $2.000 \mathrm{~kW}$ & $2.000 \mathrm{~kW}$ & $2.000 \mathrm{~kW}$ \\
\hline Rotordurchmesser & $80 \mathrm{~m}$ & $80 \mathrm{~m}$ & $90 \mathrm{~m}$ \\
\hline Nabenhöhe & $60 \mathrm{~m}$ & $100 \mathrm{~m}$ & $125 \mathrm{~m}$ \\
\hline Ertrag & $4,4 \mathrm{Mio} . \mathrm{kWh} / \mathrm{a}$ & $5,2 \mathrm{Mio} . \mathrm{kWh} / \mathrm{a}$ & $6,5 \mathrm{Mio} . \mathrm{kWh} / \mathrm{a}$ \\
\hline Volllaststunden & $2.200 \mathrm{~h} / \mathrm{a}$ & $2.600 \mathrm{~h} / \mathrm{a}$ & $3.250 \mathrm{~h} / \mathrm{a}$ \\
\hline
\end{tabular}

Anlagenzahl für die Umsetzung der Energiestrategie 2020 könnte so um 800 verringert werden (Weinhold 2009: 18).

\subsection{Landschaftsästhetische Gründe für Akzeptanzverlust}

Die baurechtliche Privilegierung von Windkraftanlagen im Außenbereich von Gemeinden ist sichtbarer Ausdruck des politischen Willens, die Energieversorgung Deutschlands sukzessive auf regenerative Energieträger umzustellen. Die maßgebenden politischen und wirtschaftlichen Akteure sehen sich jedoch massiver Kritik seitens Naturschutzverbänden und Heimatpflegevereinen ausgesetzt. Diese verweisen auf $\S 35$ Abs. 3 Nr. 5 des Baugesetzbuches, worin betont wird, dass Bauen im Außenbereich nicht zulässig ist, wenn es die „Belange des Naturschutzes und der Landschaftspflege, des Bodenschutzes, des Denkmalschutzes oder die Eigenart der Landschaft und ihren Erholungswert beeinträchtigt oder das Orts- und Landschaftsbild verunstaltet".

Zum Verständnis dieses Konflikts ist es notwendig, den Begriff Kulturlandschaft zu erläutern. Im wissenschaftlichen Sinn ist unter Kulturlandschaft die vom Menschen genutzte und dadurch umgeformte Naturlandschaft zu verstehen. In der öffentlichen Diskussion wird der Begriff jedoch oftmals auf das Bild einer vorindustriellen, bäuerlichen Landschaft reduziert und dabei die ästhetische Funktion überbewertet. Dem Erhalt des kulturhistorischen Archivs kommt im Rahmen der Kulturlandschaftspflege eine entscheidende Bedeutung zu (Dix/Schenk 2007: 819). Heimatpflegevereine kritisieren nun, dass der im ländlichen Raum historisch gewachsene Höhenmaßstab in Form der bisherigen Maßstabsbildner wie Bäume und Kirchtürme (20-35 m) durch den Ausbau von Windkraftanlagen (bis $190 \mathrm{~m}$ ) verloren geht (vgl. Abb. 5). Es wird betont, dass diese Maßstabsverluste zu einer ästhetischen Abschwächung und Verdrängung naturräumlicher sowie kultureller „Großereignisse eines Raumes“ führen (Nohl 2010: 9). Im Hinblick auf das touristische Potenzial einer Region gewinnen diese Eigenartverluste auch aus ökonomischer Perspektive an Relevanz (ARGE Monitoring PV-Anlagen 2007: 37 und 48). Darüber hinaus befürchtet man, dass eine Überformung der Kulturlandschaft durch Windkraftanlagen die

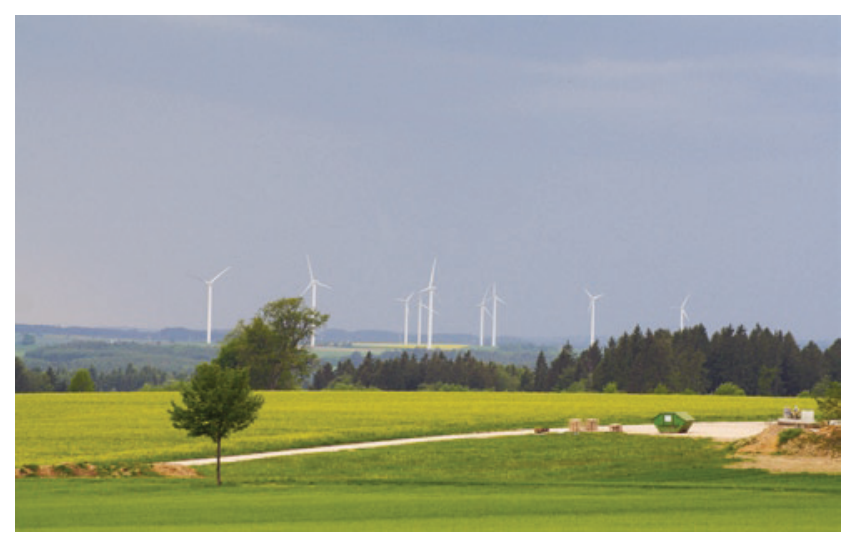

Abb. 5 Maßstabsverluste in der Kulturlandschaft durch Windkraftanlagen. (Quelle: R. Giemsa)

Grenzen zwischen den Gegenwelten einer technisch-urban geprägten Alltagswelt der Menschen und einer als naturnah empfundenen Kulturlandschaft nivelliert. Der ursprünglich ästhetisch-psychologische Effekt einer Landschaft, dem Menschen Ruhe und Gelassenheit zu vermitteln, ginge nicht zuletzt durch die permanenten Rotorbewegungen verloren (Nohl 2001: 365 ff.; Scheidler 2010: 526 f.). Belastungen des Blickfeldes können bei klarer Sicht sogar von $60 \mathrm{~km}$ entfernten Windkraftanlagen ausgehen, da exponierte Standorte wie Plateaus, Terrassen, Geländekanten und Zeugenberge gute Standortvoraussetzungen bieten (Nohl 2010: 10). Peters/Torkler/Hempp et al. (2009) führten in diesem Zusammenhang eine GIS-gestützte Landschaftsbildanalyse am Beispiel der Region Uckermark-Barnim durch, die als Grundlage für die Ausweisung von Windeignungsgebieten diente.

Störend wirkt nach Ansicht des Bayerischen Landesvereins für Heimatpflege auch die Gegensätzlichkeit zwischen der überwiegend horizontal gegliederten Landschaft und den vertikal ausgerichteten Windkraftanlagen (Horizontverschmutzung). Visuelle Beeinträchtigungen treten sogar nachts auf, denn der von künstlichem Licht bis dato relativ unbeeinflusste ländliche Raum erhält aus Gründen der Flugsicherheit durch die gleichmäßig aufgereihten Lichtsignale eines Windparks eine weithin sichtbare Quelle der Lichtver- 
schmutzung. Akustische Störungen rühren aus der Überlagerung landschaftstypischer Geräusche wie das Plätschern eines Baches, das Zwitschern von Vögeln und das Rauschen von Bäumen durch die Rotorgeräusche von Windkraftanlagen (Nohl 2010: 11 f.).

Die neueste Generation von Windkraftanlagen mit hohen Wirkungsgraden und großen Nabenhöhen rücken nun auch den Flächenstaat Bayern, der mit rund 400 Anlagen zu den Schlusslichtern in Deutschland hinsichtlich der installierten Windleistung zu zählen ist (vgl. Abb. 6), in den Fokus der Windbranche. Es ist davon auszugehen, dass dort in den kommenden Jahren etwa 1500 Windkraftanlagen errichtet werden. Ansässige Bürgerinitiativen haben bereits Kampagnen gestartet, um die bayerische Kulturlandschaft vor einer „Verspargelung" zu bewahren. Befürchtet werden negative Auswirkungen auf den Tourismus, denn die Errichtung mehrerer, an der Rotorspitze bis zu $190 \mathrm{~m}$ hoher Windkraftanlagen zwischen Ammersee und Starnberger See würde zu einem ungewohnten Landschaftsbild führen (Sebald 2010: 42).

Die Windbranche ist sich der Tatsache bewusst, dass eine Energiewende nur dann erfolgreich vollzogen werden kann, wenn sie auf dem Fundament einer breiten Akzeptanz in der Bevölkerung gründet. Bei Windprojekten ist dies jedoch nicht immer gewährleistet, da mit Hilfe einer gerichtlichen Entscheidung Vorhaben auch gegen eine kommunale Mehrheit durchgesetzt werden können (ARGE Monitoring PV-Anlagen 2005: 38). Eine offensive Informationspolitik sowie Aufklärung über Möglichkeiten der Teilhabe an einem Energieversorgungssystem, das auf endogene Potenziale setzt, regionale Wirtschaftskreisläufe ankurbelt und die Unabhängigkeit von überregionalen Versorgern fördert, ist hierbei ein bedeutender Faktor. Entscheidend ist jedoch, inwieweit es gelingen wird, die Dichotomie zwischen dem Erhalt einer attraktiven Kulturlandschaft und dem Ausbau von erneuerbaren Energien aufzulösen. Hierzu gibt es bereits ausgereifte Konzepte und gelungene Vorzeigeprojekte, die den Interessenkonflikt zwischen den bewahrenden Kräften der Heimatpfleger, den hohen Ansprüchen der Naturschützer und dem am Fortschritt orientierten Willen von Politik und Wirtschaft auszugleichen versuchen. Angesichts dieser Konfliktlage erscheint es notwendig, verstärkt gesellschaftliche Debatten über die Grenzen und Möglichkeiten von erneuerbaren Energien auf kommunaler Ebene zu führen. Dabei müssen die Fehlentwicklungen der letzten Jahre thematisiert werden und als Erfahrungswerte in neue Vorzeigeprojekte einfließen. Dadurch könnte die Akzeptanz für einen weiteren Ausbau von erneuerbaren Energien im ländlichen Raum wieder erhöht werden. Gelingt dies nicht, so ist eine stärkere Fokussierung der Windbranche auf Großprojekte im Offshore-Bereich zu erwarten.

\subsection{Energie aus dem Meer}

\subsubsection{Europa setzt auf Offshore-Windenergie}

Das Ausweichen der Windbranche auf schwierig zu erreichende Offshore-Standorte bringt nicht nur Nachteile mit sich, denn Windparks auf hoher See generieren im Durchschnitt $50 \%$ mehr Ertrag als jene an Land. Dies liegt daran, dass die Windgeschwindigkeiten über dem Meer aufgrund der geringeren Reibung höher sind und der Wind konstant weht. Diese Standortvorteile spiegeln sich in einem verstärkten Ausbau wider. Global betrug der Zuwachs im Jahr 2008373 MW, 2009577 MW und für 2010 rechnet man
Abb. 6 Installierte Windleistung auf Länderebene. (Quelle: Eigene Darstellung nach Bundesverband WindEnergie 2010: 1 f.)

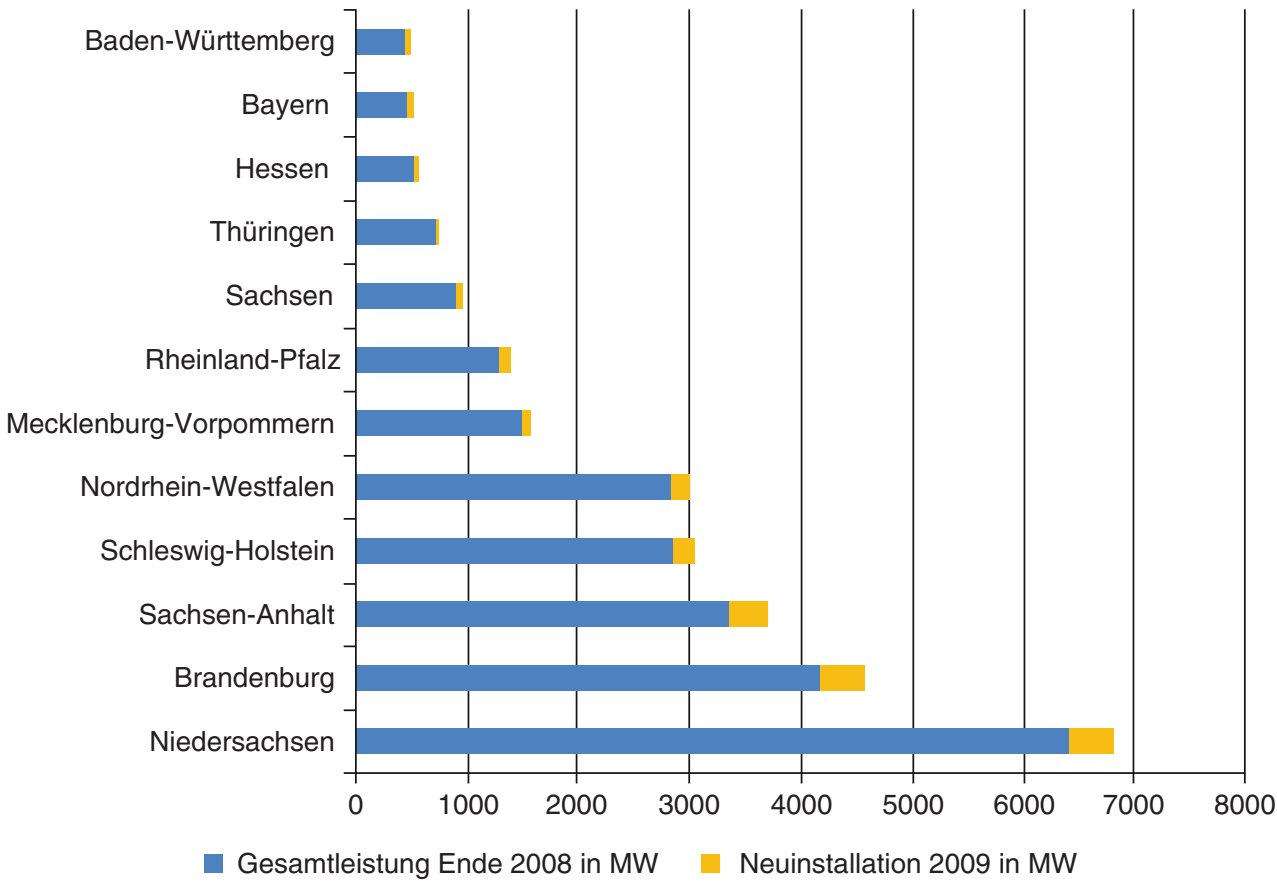


sogar mit einer zusätzlich installierten Offshore-Leistung von einem Gigawatt. Der Europäische Windenergieverband (EWEA) geht von einem jährlichen Wachstum von $28 \%$ bis zum Jahr 2020 aus (Lessner 2010a: 18). Dabei nimmt Großbritannien eine herausragende Stellung ein. Bereits 2009 waren dort $600 \mathrm{MW}$ auf Offshore-Standorten installiert und $1700 \mathrm{MW}$ befanden sich bereits im Bau. Jedoch sieht es nicht danach aus, als könnten die ambitionierten Ziele, bis 2020 eine $O f f$ shore-Leistung von $29 \mathrm{GW}$ zu installieren, erreicht werden. Dies liegt daran, dass für ganz Europa derzeit nur neun Schiffe für die Verlegung von Seekabeln zur Verfügung stehen. Allein Großbritannien bräuchte jedoch zur Umsetzung seiner Energiestrategie 58 Schiffe, um die Windparks untereinander und mit dem Festland verbinden zu können. Von Vorteil ist, dass der Ausbau der britischen Offshore-Windenergie auf einer großen gesellschaftlichen Akzeptanz gründet. Die Genehmigungswahrscheinlichkeit von Projekten in unmittelbarer Nähe zur Küste - auch innerhalb der 12-Seemeilen-Zone - ist vergleichsweise hoch (Kratzsch 2009: 32). Im Gegensatz dazu stehen die starken räumlichen Einschränkungen innerhalb der Deutschen Bucht. Einerseits ist der Nationalpark Wattenmeer generell von Bauvorhaben freizuhalten. Darüber hinaus verhindert die der deutschen Küste vorgelagerte Inselkette schon aus natürlichen Gründen einen küstennahen Ausbau. Die bis dato geringe Dynamik bei der Initiierung von Offshore-Projekten im Vergleich zu anderen EU-Staaten ist unter anderem darauf zurückzuführen.

\subsubsection{Deutschlands verspäteter Einstieg in den Offshore-Bereich}

Mit der Fertigstellung des Windparks „Alpha Ventus“ im Jahr 2009 sowie seines Netzanschlusses 2010 nahm der Ausbau der Offshore-Windenergie auch in Deutschland konkret Gestalt an (Gille 2010: 24 ff.). Dabei wurden 12 Windkraftanlagen mit einer Nabenhöhe von jeweils $90 \mathrm{~m}$, einer Gesamtleistung von $60 \mathrm{MW}$ und einem Investitionsvolumen von 250 Mio. $€$ rund $45 \mathrm{~km}$ nördlich der Insel Borkum in $30 \mathrm{~m}$ Meerestiefe installiert. Die Netzanbindung erfolgt über eine $60 \mathrm{~km}$ lange, im Untergrund versenkte und über die Insel Norderney verlaufende Kabeltrasse (vgl. Abb. 7). Das Projekt dient in erster Linie dazu, Erfahrungen mit Windkraftanlagen unter Offshore-Bedingungen zu sammeln (Alpha Ventus 2010: 6 ff.). Nach kurzer Laufzeit konnten bei „Alpha Ventus“ bereits schwerwiegende Ero-
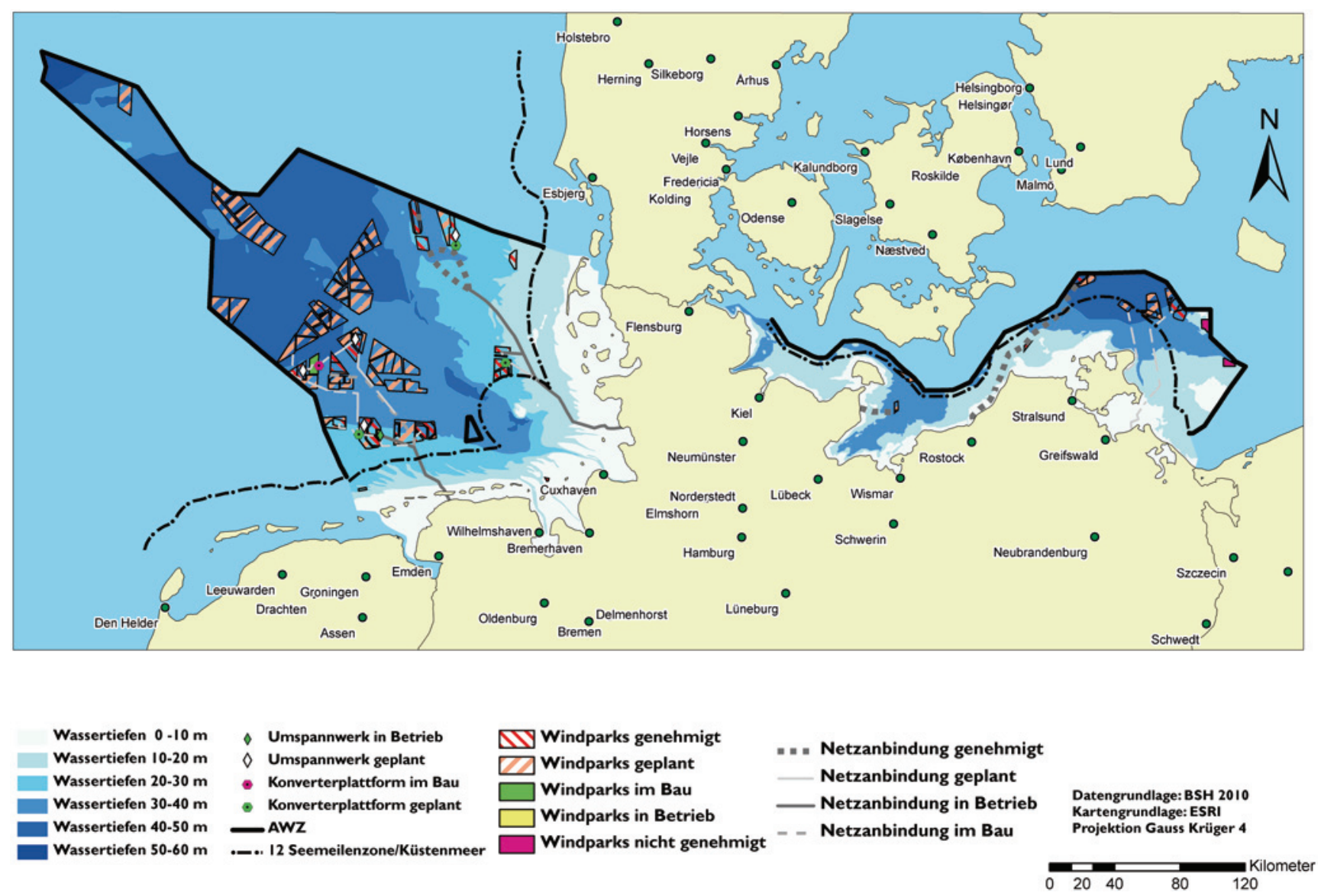

Abb. 7 Geplante und genehmigte Windparks in der Ausschließlichen Wirtschaftszone (AWZ). (Quelle: Eigene Darstellung nach Bundesamt für Seeschifffahrt und Hydrographie 2009: 1 f.) 
sionen (Kolkentstehung) an den Gründungen der Windkraftanlagen festgestellt werden (Reuning 2010: 24). Eine kostengünstige Variante zur Stabilisierung von Fundamenten besteht nun darin, größere Steine oder Sandsäcke aus Geotextilien im Umkreis von Gründungen anzuhäufen. Darüber hinaus wird diskutiert, ob Kolkschutz - d. h. konstruktive Maßnahmen zur Vermeidung von Eintiefungen in Gewässersohlen - durch die Verwendung größerer Fundamente garantiert werden kann.

Bis 2030 sollen die Offshore-Leistung in der Ausschließlichen Wirtschaftszone Deutschlands (AWZ) auf 25.000 MW gesteigert und $85 \mathrm{Mrd}$. $\mathrm{kWh}$ Strom pro Jahr produziert werden (vgl. Bard 2006: 54). Der Kampf um lukrative OffshoreStandorte innerhalb der Ausschließlichen Wirtschaftszone wird dabei allein zwischen den großen Energieversorgern ausgefochten (vgl. Abb. 7). Das Investitionsvolumen der Bundesregierung für den geplanten Ausbau der OffshoreWindenergie beträgt etwa $50 \mathrm{Mrd}$. $€$. Neben der Förderung von Offshore-Windparks sowie deren Netzanbindung fließen die Gelder in die maritime Installations-, Service- und Dienstleistungsbranche. Darüber hinaus wird die Hafeninfrastruktur den neuen Gegebenheiten angepasst. Abgesehen von der finanziellen Unterstützung versucht die Bundesregierung den Ausbau durch Änderungen von gesetzlichen Rahmenbedingungen zu erleichtern. Neben dem am 17. Dezember 2006 in Kraft getretenen Infrastrukturplanungs- beschleunigungsgesetz lag das Augenmerk auf der Ausdehnung des Vergütungszeitraumes bzw. einer Verzögerung der Degression (Bundesministerium für Umwelt, Naturschutz und Reaktorsicherheit 2007: 10).

\subsubsection{Nutzungsdruck auf hoher See}

Betrachtet man die Integration der Offshore-Windenergie in den Raumordnungsplan der Ausschließlichen Wirtschaftszone, so ist zu erkennen, dass die Ressource Raum selbst auf hoher See ein wertvolles Gut ist (vgl. Abb. 8). Neben Vorranggebieten für Seeschifffahrt, Fauna-Flora-HabitatGebieten (FFH) und Vogelschutzgebieten verbleiben nur noch wenige Flächen für den Ausbau der Windenergie in Küstennähe. Eine Orientierung auf weiter entfernte Standorte ist jedoch trotz höherer Transport- und Wartungskosten möglich, denn Nord- und Ostsee weisen langsam abfallende Böden auf. Mit den derzeit zur Verfügung stehenden Fundamenten (z. B. Tripod) sind Windprojekte bis zu einer Meerestiefe von etwa $40 \mathrm{~m}$ lukrativ. Im Vergleich zu den schnell abfallenden Uferzonen des Atlantiks und Mittelmeeres bietet die Deutsche Bucht damit ein großes Standortpotenzial. Der beispielsweise im Jahr 2002 vom dänischen Unternehmen Elsam (heute DONG Energy) errichtete Offshore-Windpark „Horns Rev“, mit 80 Windkraftanlagen und einer installierten Leistung von $160 \mathrm{MW}$,
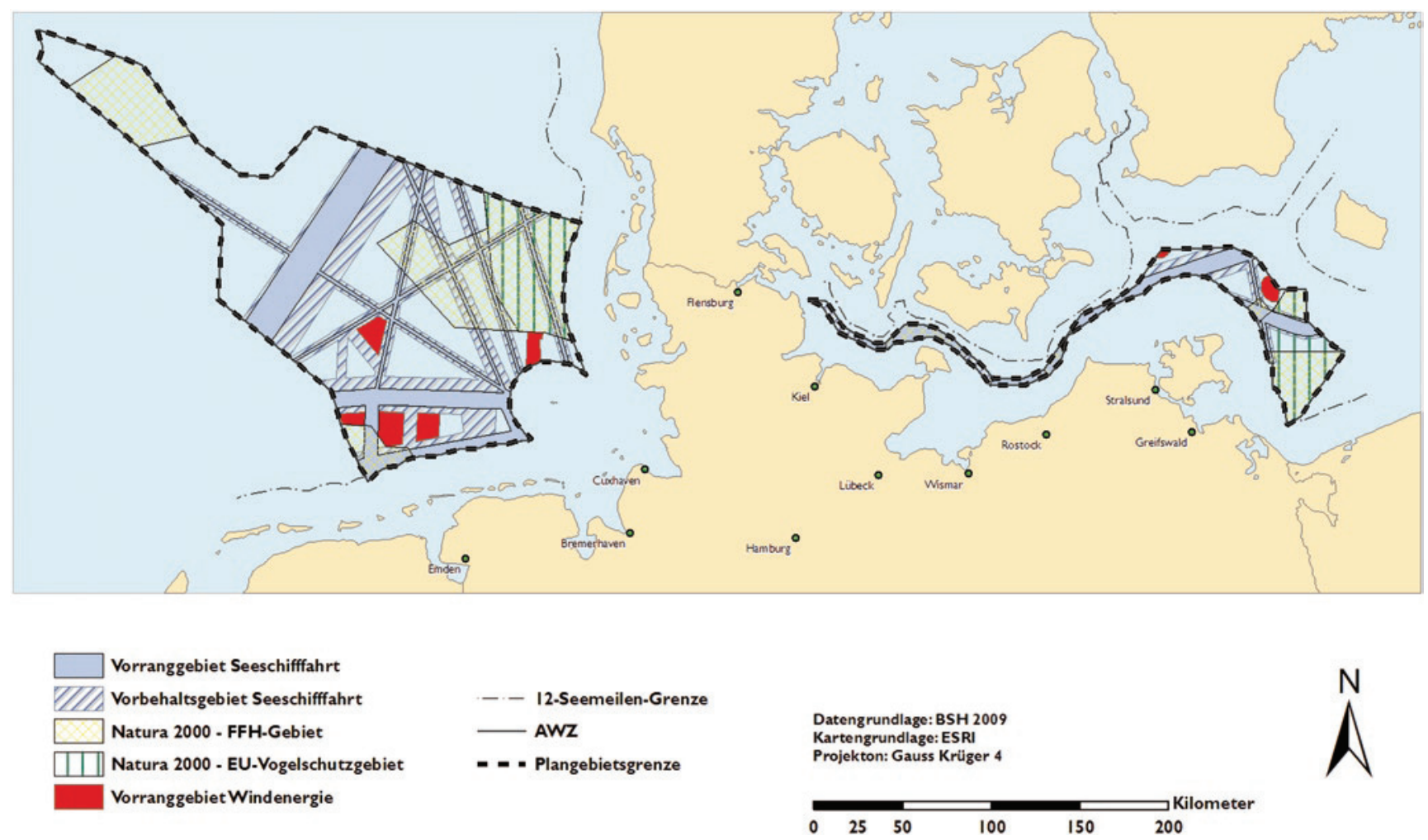

Abb. 8 Integration der Offshore-Windenergie in den Raumordnungsplan der Ausschließlichen Wirtschaftszone (AWZ). (Quelle: Eigene Darstellung nach Bundesamt für Seeschifffahrt und Hydrographie 2009: $1 \mathrm{f}$.) 
wurde in einer Entfernung von 14 bis $20 \mathrm{~km}$ vom Festland bei einer Wassertiefe von nur 6-14 m errichtet. Die Größendimensionen des Windparks verdeutlichen auch, warum die Flächennutzungskonkurrenz innerhalb küstennaher Areale der Ausschließlichen Wirtschaftszone durch Offshore-Windparks erheblich verstärkt wird. Aus Gründen der Windabschattung sowie raumordnungsrechtlicher Vorgaben beträgt der Abstand zwischen den einzelnen Anlagen 560 m. Damit nimmt „Horns Rev“ eine Fläche von 20 km $^{2}$ ein (vgl. Elsam 2002). Eine kontinuierliche Erhöhung der installierten Offshore-Leistung ist aus diesem Grund nur realistisch, wenn der zukünftige Ausbau weiter in die Nordsee vordringen kann. Der Einsatz schwimmender OffshoreFundamente stellt dabei einen geeigneten Ansatz dar (Spar Buoy-, Tension Leg Platform- und Barge-Prinzip). Dadurch könnten küstenferne Standorte mit Meerestiefen von bis zu 700 m für WKA erschlossen werden (Lessner 2010a: 20 f.). Da die schwimmenden Fundamente über Seile am Boden fixiert werden müssen, spielt die Meerestiefe bei der Standortplanung nach wie vor eine Rolle. Von Vorteil ist, dass bezüglich dieser technologischen Herausforderung auf langjährige Erfahrungen aus der Offshore-Ölindustrie zurückgegriffen werden kann.

Neben Windkraftanlagen drängen mittlerweile neue regenerative Technologien in den Offshore-Bereich vor. Der Einfallsreichtum von Ingenieuren, die Kraft des Meeres in Strom umzuwandeln, ist dabei vielversprechend. Wellen-, Osmose-, Meeresströmungs- und Meereswärmekraftwerke sind jedoch noch weit von der industriellen Reife entfernt. Lediglich Gezeitenkraftwerke - die für den Standort Deutschland nicht in Frage kommen - sind derzeit in der
Lage, regional einen nennenswerten Beitrag zur Stromproduktion zu leisten (Bard 2006: 56 ff.; Lessner 2009: 78).

\section{Räumliche Neuorientierung der Solarbranche}

\subsection{Technisierung der Kulturlandschaft}

Im Gegensatz zur Windenergie erfassen die Privilegierungstatbestände des $\S 35$ Abs. 1 BauGB nicht die Errichtung von Photovoltaik-Freiflächenanlagen. Die Steuerung von Projekten erfolgt über die kommunale Bauleitplanung, die nicht zuletzt grundlegend für den Anspruch auf Vergütung seitens des Netzbetreibers ist. Neben dem Bebauungsplanverfahren greifen Baugenehmigungsverfahren sowie naturschutzrechtliche Ausnahmegenehmigungen (Bundesministerium für Wirtschaft und Technologie 2010: 4 ff.). Generell treffen Photovoltaik-Freiflächenanlagen auf eine größere Akzeptanz in der Bevölkerung als Windkraftanlagen (vgl. Abb. 9), denn sie bedürfen eines Bebauungsplanes und sind damit von der kommunalen Mehrheit abhängig. Problematisch ist, dass die schlechten Erfahrungen der Bevölkerung mit Windprojekten auch zu großer Skepsis gegenüber Solarprojekten geführt haben. Bürgerinitiativen, die ursprünglich aus dem Widerstand gegen Windkraftanlagen entstanden sind, bleiben teilweise auch gegenüber Solarparks formiert (ARGE Monitoring PV-Anlagen 2005: $38 \mathrm{f}$. und 41).

Aufgrund der geringen Strahlungsenergie in Deutschland von etwa $300-600 \mathrm{~W} / \mathrm{m}^{2}$ müssen Photovoltaik-Freiflächenanlagen - um einen entsprechenden Energieertrag liefern zu können - großflächig angelegt sein. Dabei führen Uniformi-
Abb. 9 Akzeptanz von Stromerzeugungsanlagen in räumlicher Nähe des Wohnortes. (Quelle: Eigene Darstellung nach Forsa 2009: 7)

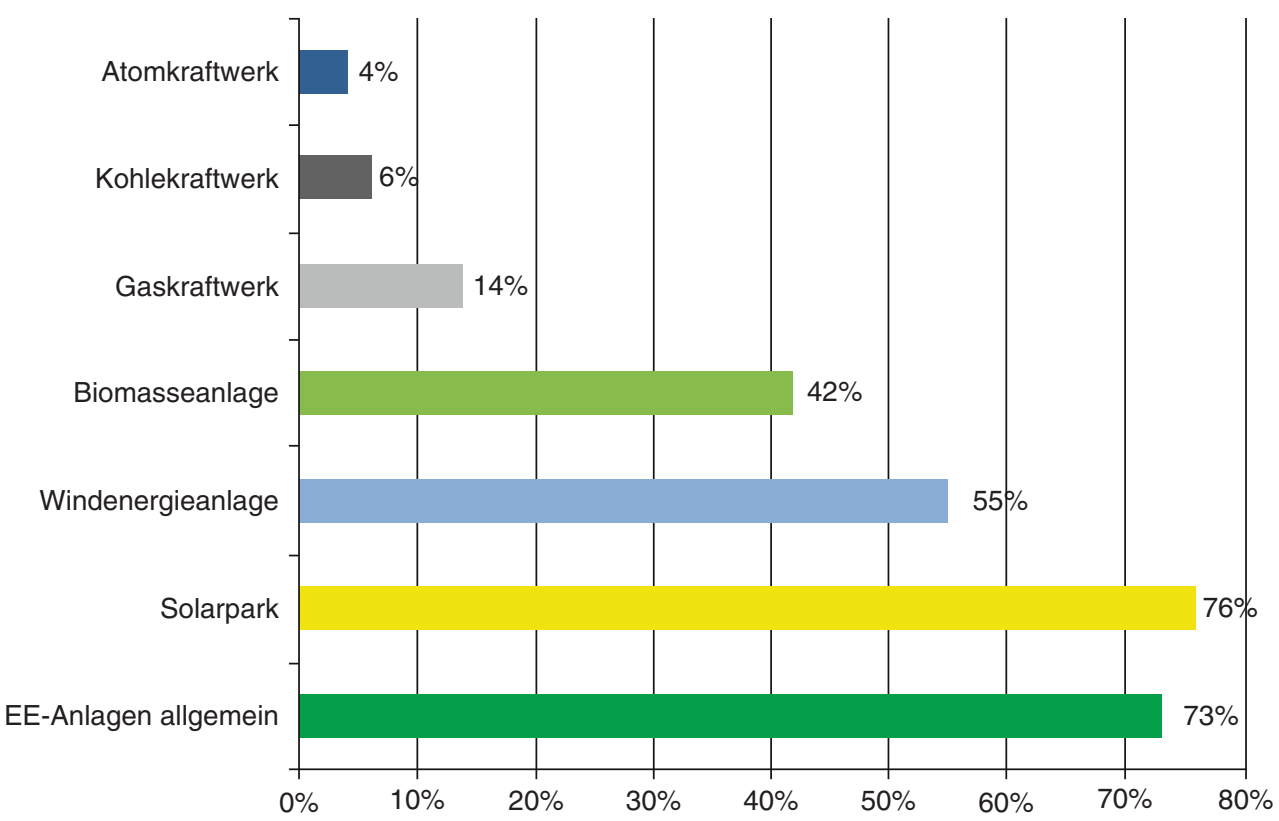


tät und die Verwendung landschaftsfremder Baumaterialien zu einem sichtbaren Eingriff in die Kulturlandschaft. Die optische Wirkung hängt im konkreten Fall von der jeweiligen Farbgebung der Bauteile, der Reflexionseigenschaften, der Lage in der Horizontlinie, der damit verbundenen Silhouettenwirkung sowie vom Sonnenstand und Bewölkungsgrad ab. Im Nahbereich der Anlagen sind technische Einzelheiten gut erkennbar und ziehen die Aufmerksamkeit des Betrachters an (ARGE Monitoring PV-Anlagen 2007: $32 \mathrm{f}$ ). Bei Anlagenhöhen von bis zu 3,5 m wird darüber hinaus die Aussicht für Anwohner in der näheren Umgebung verbaut (ARGE Monitoring PV-Anlagen 2005: 38). Bei größeren Entfernungen sind Freiflächenanlagen gut zu erkennen, denn oftmals hebt sich ihre homogene farbliche Gestaltung deutlich von der umgebenden, heterogen strukturierten Landschaft ab. Speziell bei Anlagen an weit einsehbaren, exponierten Standorten (z. B. Hanglage) ergeben sich sichtbare Veränderungen im Landschaftsbild. Durch Reflexblendungen kann es beim Betrachter sogar zu vorübergehenden Funktionsstörungen des Auges kommen, wobei Studien diesen Aspekt für vernachlässigbar erachten (ARGE Monitoring PV-Anlagen 2007: 35). Bedenklich ist es, wenn Photovoltaik-Anlagen in Folge ihrer Einzäunung der Landschaft hochwertige Lebensräume entziehen und als räumliche Barrieren wirken. Dadurch können gewachsene Wegebeziehungen zerschnitten werden, so dass es zum Eingriff in faunistische Funktionsbeziehungen und zur Fragmentierung von Habitatstrukturen kommen kann (ARGE Monitoring PV-Anlagen 2007).

Da Freiflächenanlagen grundsätzlich an eine Siedlung angebunden werden müssen, ist auf die Verhältnismäßigkeit zwischen der Größe eines Dorfes und der des Solarparks zu achten (Bayerisches Staatsministerium des Innern 2009: 2). Mit größerer Nähe einer Freiflächenanlage zu einer Siedlung geht jedoch die Akzeptanz in der ansässigen Bevölkerung zurück. Dabei spielen die flächenhaften Ausmaße der Anlagen in der Bewertung eine untergeordnete Rolle. Eine ablehnende Haltung in der Bevölkerung entspringt nur in seltenen Fällen aus der Skepsis gegenüber einem kon kreten Projekt. Vielmehr wird befürchtet, dass durch die Zustimmung zu einem Vorhaben ein Präzedenzfall geschaffen wird, der weiteren Vorhaben den Weg ebnet. Generell besitzen Solarparks in industriell geprägten Regionen eine höhere Genehmigungswahrscheinlichkeit als in ländlichen Räumen (ARGE Monitoring PV-Anlagen 2005: 40).

\subsection{Landschaftsarchitektur als Mittler}

Eine Lösungsstrategie, der wachsenden Skepsis gegenüber Solarprojekten begegnen zu können, besteht in der räumlichen Integration von Solarparks. Dabei können Landschaftsarchitekten als Mittler zwischen Heimatpflegevereinen, Naturschutzverbänden und Industrie fungieren, denn ihre
Aufgabe bestand von jeher darin, neue Landschaftselemente möglichst ästhetisch in vorhandene räumliche Strukturen einzubetten. Bei der Errichtung von Photovoltaik-Freiflächenanlagen muss zur Kenntnis genommen werden, dass Blockstrukturen nur schwer in die Formenvielfalt gewachsener Kulturlandschaften zu integrieren sind. Kleinteilige Parzellenstrukturen lassen sich hingegen gut an den individuellen Charakter einer Landschaft anpassen und sorgen darüber hinaus für eine größere Biodiversität (Peters 2010: 26). Entscheidend ist, dass die Anlagen der Formenvielfalt der parzellierten landwirtschaftlichen Nutzflächen angepasst werden und den Verlauf von Verkehrswegen respektieren. Optisch attraktiv wirken vor allem jene Solarparks, die sich aus mehreren, unterschiedlich geformten, jedoch aufeinander abgestimmten Teilflächen zusammensetzen (vgl. Abb. 10). Die Einhaltung von Abstandsflächen zwischen den einzelnen Solarfeldern sorgt für Auflockerung sowie Durchlässigkeit und verhindert dadurch eine optische Dominanz in der Kulturlandschaft. Zur weiteren, möglichst naturnahen Integration von Solarparks dient deren Einfriedung mittels lockerer Baumreihen aus Hecken mit Obstbäumen, Eichenhainen sowie Böschungen mit Feldgehölzen. Zusätzlich werden Ausgleichsflächen geschaffen, innerhalb derer sich nach kurzer Zeit eine Artenvielfalt etabliert, die jene auf den zuvor landwirtschaftlich genutzten Flächen übersteigt. Nicht zuletzt stellen die von den Solarmodulen besetzten Areale einen geeigneten Standort für die Beweidung durch Schafe dar (vgl. Wartner 2010).

Entsprechend der ermittelten Potenziale der Agentur für Erneuerbare Energien müssten bis 2020 zusätzlich 8800 ha für die Errichtung von Freiflächenanlagen erschlossen werden (vgl. Abb. 3). Jedoch wurden die räumlichen Möglichkeiten der Photovoltaik-Branche durch die Novellierung des EEG 2010 und die damit einhergehende Verringerung der Einspeisevergütung um $16 \mathrm{ct} / \mathrm{kWh}$ sowie den Ausschluss von Ackerflächen aus der Vergütungsstruktur stark eingeschränkt (Bundesministerium für Umwelt, Naturschutz und

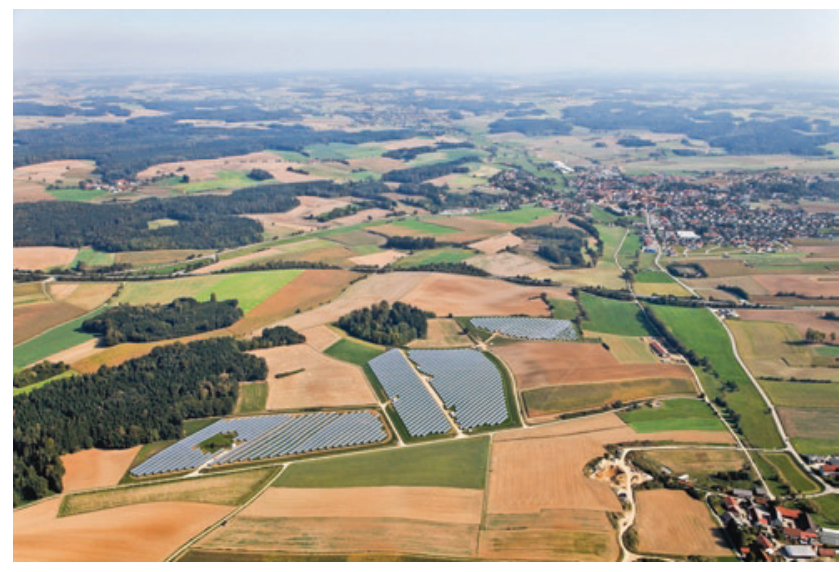

Abb. 10 Integration von Photovoltaik-Freiflächenanlagen in die Kulturlandschaft. (Quelle: K. Leidorf) 
Reaktorsicherheit 2010: 1 f.). Zwar treffen gebäudeintegrierte Lösungen auf große Akzeptanz in der Bevölkerung, dennoch werden diese Flächenpotenziale nicht ausreichen, um eine rasche Energiewende in Deutschland herbeiführen zu können. Mit Hilfe von Dachflächen werden maximal $20 \%$ des deutschen Strombedarfs gedeckt (EuPD Research 2008: 29).

\subsection{Nutzung von Konversionsflächen}

Aus ökologischer Sicht muss die Novellierung des EEG 2010 kritisch hinterfragt werden, da mit der energetischen Nutzung von Ackerflächen die Verpflichtung einherging, die Flächen in Grünland - das gewöhnlich zu einer Verringerung der Bodenerosion, zu einer besseren Aufnahmefähigkeit von Niederschlagswasser und zu einer Erhöhung der Artenvielfalt führt - umzuwandeln. Die räumlichen Restriktionen werden zwangsläufig zu einer verstärkten Nutzung von Konversionsflächen und damit zu einem verringerten Wettbewerb um qualitativ hochwertige Flächen führen. Die Umwidmung von ehemaligen Militärflächen zu Gunsten des Ausbaus der Solarenergie ist in Deutschland bereits in vollem Gange. 2008 wurde auf dem ehemaligen Militärflughafen im Energiepark Waldpolenz - östlich von Leipzig - eine Freiflächenanlage mit einer Leistung von $40 \mathrm{MW}$ errichtet. Ein Jahr später ging der Solarpark Lieberoser Heide (Brandenburg) mit einer installierten Leistung von $53 \mathrm{MW}$ und einer Grundfläche von 163 ha ans Netz. Zuvor wurde der ehemalige sowjetische Truppenübungsplatz auf einer Fläche von 400 ha von Munition und chemischen Altlasten befreit (vgl. juwi solar GmbH 2010). Die Solarenergie bietet folglich die Gelegenheit, den Aufbau einer umweltfreundlichen Energieversorgung mit einer Renaturierung von militärischen Liegenschaften zu kombinieren. Dennoch muss zur Kenntnis genommen werden, dass nicht alle Konversionsflächen Deutschlands für den Ausbau zur Verfügung stehen. Oftmals bildeten sich auf den Flächen schützenswerte Trocken- und Magerrasenbiotope sowie Rückzugsgebiete für bedrohte Vogelarten aus (ARGE Monitoring PV-Anlagen 2007: 46).

Mittlerweile gibt es auch Bemühungen, die Photovoltaik im Offshore-Bereich einzusetzen. Nachdem an der Fachhochschule Nordhausen in Thüringen bereits Versuche mit schwimmenden Photovoltaik-Anlagen durchgeführt wurden, ist nun in Planung, auf einem Kiessee (Konversionsfläche) in Babenhausen (Hessen) das erste kommerzielle Offshore-Photovoltaik-Kraftwerk mit einer Leistung von 6,2 MW zu errichten (Becker 2010: 58 f.).

\subsection{Wüstengebiete als alternative Standorte}

Angesichts der starken räumlichen Einschränkungen stellt sich die Suche nach Standorten im Ausland, die sich durch eine geringe Flächennutzungskonkurrenz auszeichnen, als eine weitere Option dar. Dabei kommen die großen technologischen Fortschritte im Bereich solarthermischer Kraftwerke zu Hilfe, die eine Erschließung von Standortpotenzialen in den Wüstengürteln der Erde als lukrativ erscheinen lassen (Bosch 2010: 1). Mit dem Projekt „Desertec“ wagt man seitens der Industrie nun den Schritt, den vom „Club of Rome“ bereits lang gehegten Traum von der Nutzung der Sonnenenergie in den Wüstengebieten der Erde in die Tat umzusetzen. Dabei bestehen die zentralen Herausforderungen darin, Strahlungsenergie aus den Wüstengürteln der Erde in wirtschaftlich nutzbare Energie umzuwandeln und diese, mit Hilfe von Hochspannungs-Gleichstrom-Übertragungsleitungen, bis zu den $3000 \mathrm{~km}$ entfernten Verbrauchern zu transportieren. Das Energiepotenzial ist erstaunlich, denn in sechs Stunden empfangen die Wüstengebiete der Erde mehr Sonnenenergie, als die Menschheit innerhalb eines Jahres verbraucht (Desertec Foundation 2010: 3). Dieses Potenzial lässt sich durch die bereits ausgereiften konzentrierenden solarthermischen Systeme erschließen. Im Mittelpunkt des Interesses stehen hierbei Parabolrinnenkraftwerke, Fresnelkollektoren, Dish-Stirling-Anlagen und Solartürme, die in Kombination mit Salzspeichern eine grundlastfähige Stromproduktion ermöglichen. Darüber hinaus besteht die Absicht, die Abwärme der Anlagen für den Betrieb von Meerwasserentsalzungsanlagen zu nutzen (Deutsches Zentrum für Luft- und Raumfahrt 2007: 97 ff.).

\section{Einseitige Ausrichtung des Energiepflanzenbaus}

Die Energiegewinnung mittels Biomasse hat bereits seit Jahren das schlechteste Image unter den erneuerbaren Energien (vgl. Abb. 9). Betreiber von Biogasanlagen sehen sich einer massiven Gegenbewegung seitens Bürgerinitiativen, Heimatpflegevereinen und Naturschutzverbänden ausgesetzt. Kanning/Buhr/Steinkraus (2009: 144) führen diese Irritationen unter anderem darauf zurück, dass zwischen den einzelnen Biomassenutzungspfaden unzureichend differenziert wird und speziell im Hinblick auf die ökologischen und räumlichen Dimensionen des Biogaspfades eine große Forschungslücke klafft. Ursprünglich geht der Konflikt auf die Novellierung des EEG im Jahr 2004 zurück. Mit der damaligen Einführung des Bonus für nachwachsende Rohstoffe (Nawaro), die den Energieträger Silomais als wichtigstes Substrat bei der Versorgung von Biogasanlagen etablierte, gingen aus ökologischer Sicht bedenkliche Veränderungen in der Kulturlandschaft wie beispielsweise der Verlust an Biodiversität einher (Bosch 2008: 8 f.; Bosch/Peyke 2008: 454). Der Anbau von Silomais hat sich in Deutschland seit 2004 von 1.248 .000 ha auf 1.647 .000 ha, folglich um $32 \%$ erhöht. Das Deutsche Maiskomitee führt diesen Anstieg auf den Substratbedarf von Biogasanlagen zurück. Energiemais wird derzeit auf etwa 380.000 ha angebaut. In 15 Land- 
kreisen, beispielsweise im Landkreis Rotenburg (Wümme), nimmt der Anbau von Silomais mittlerweile einen Anteil von über $50 \%$ an der Ackerfläche ein (Lessner 2010b: 77). Aufgrund der zunehmenden „Vermaisung“ der Kulturlandschaft plädiert der Naturschutzbund Deutschland (NABU) in einem Zehn-Punkte-Papier für eine naturverträgliche und damit räumlich stark eingeschränkte Biogasproduktion. Damit verbunden ist die Forderung, den Anteil von Silomais an der Substratversorgung einer Biogasanlage auf $50 \% \mathrm{zu}$ begrenzen. Die Umsetzung dieser Forderung würde jedoch die Wirtschaftlichkeit vieler Biogasanlagen in Frage stellen, denn der hohe Biomasseertrag und der daraus resultierende hohe Gasertrag pro Hektar qualifizieren den Silomais nach wie vor zum wichtigsten Substrat. Seine Bedeutung spiegelt sich in der jährlichen Ausbeute an Kilowattstunden pro Hektar wider. Durchschnittlich erreichen Energiepflanzen einen Wert von $33.000 \mathrm{kWh} / \mathrm{ha} / \mathrm{Jahr}$ (vgl. Plank 2004: 8). Im Falle von Silomais sind durchschnittlich $50.000 \mathrm{kWh} / \mathrm{ha} / \mathrm{Jahr}$ zu erwarten, auf optimalen Silomaisstandorten ist mit den neuesten Sorten sogar ein Energieertrag von $110.000 \mathrm{kWh} /$ ha/Jahr möglich (Fachverband Biogas 2005: 4). Berechnet man den Wirkungsgrad der Blockheizkraftwerke von etwa $35-40 \%$ mit ein, so lassen sich aus einem Hektar Silomais 17.000-20.000 kWh Strom erzeugen. Damit kann der Strombedarf von fünf Haushalten gedeckt werden bzw. ein Erdgas-Auto $70.000 \mathrm{~km}$ weit fahren (vgl. Fachverband Biogas 2005: 7). Davon abgesehen ist Silomais für die in der Biogasanlage eingesetzten Bakterien leicht verdaulich. Dies garantiert eine hohe Gasausbeute und Prozessstabilität (Ott 2006: 48). Vergleicht man diese Energieausbeute jedoch mit jener von Windkraft und Photovoltaik, so offenbaren sich die Nachteile des Energiepflanzenbaus. Für die Produktion von $1 \mathrm{GWh}$ Endenergie benötigt man bei Biogasanlagen 102 ha, bei Windkraftanlagen 5,7 ha und bei PhotovoltaikAnlagen nur 4,4 ha (Peters 2010: 17).

Forschung und Entwicklung stehen in der Verantwortung, neben Silomais neue, ertragreiche Energiepflanzen zu etablieren und ökologisch unbedenkliche Energiefruchtfolgen auch aus ökonomischer Sicht möglich zu machen. Im Mittelpunkt des Interesses stehen dabei massenwüchsige Pflanzen wie Sudangras (Sorghum sudanense) und Chinaschilf (Miscanthus sinensis) (Fachagentur Nachwachsende Rohstoffe 2007: 20 f.). Eine weitere Forderung des Naturschutzbundes Deutschland (2010) ist ein absoluter Verzicht seitens der Landwirtschaft darauf, Grünland zu Gunsten neuer Standorte für Energiepflanzen umzubrechen. Überhaupt seien im Rahmen der Biogasproduktion ökologisch sensible Standorte (z. B. starke Hangneigung) zu meiden sowie ökologische Ausgleichsflächen (z. B. Extensivgrünland, Feldgehölze, Blühstreifen) in Höhe von $10 \%$ der Betriebsfläche nachzuweisen. Angesichts der von der Agentur für Erneuerbare Energien formulierten Zielsetzungen zum Ausbau von erneuerbaren Energien ist jedoch zu erwar- ten, dass sich der Konflikt zwischen Naturschutzverbänden und Anlagenbetreibern noch verschärfen wird, denn um bis 2020 jährlich 54,3 Mrd. kWh Strom, 150,3 Mrd. kWh Wärme und 111,3 Mrd. kWh Kraftstoff liefern zu können, müsste der Bioenergie ein Anteil von 21,9\% an der landwirtschaftlich genutzten Fläche (3,7 Mio. ha) zugestanden werden (vgl. Abb. 3).

Für einen Ausgleich widerstrebender Nutzungsinteressen bedarf es einer adäquaten Steuerung der Raumnutzung. Es besteht die Auffassung, dass mit dem Regionalplan bereits ein ausreichendes Instrument zur Verfügung steht. Grundsätzlich sind die räumlichen Unterschiede zwischen dem Nahrungs- und Futtermittelanbau auf der einen sowie dem Biomasseanbau für energetische Zwecke auf der anderen Seite marginal. Folglich ließe sich der Anbau von Energiepflanzen über die Ausweisung von Vorrang- und Vorbehaltsgebieten für die Landwirtschaft steuern. Auf eine gesonderte Ausweisung von Vorrang- und Vorbehaltsgebieten für den Biomasseanbau kann daher verzichtet werden (Bundesamt für Bauwesen und Raumordnung 2010: 10). Die konkrete Verortung einer Bioenergieanlage wird schließlich durch das Bundesimmissionsschutzgesetz (BImSchG), das Baurecht (BauGB) und das Gesetz über die Umweltverträglichkeitsprüfung gesteuert.

\section{Kombikraftwerke und Partizipation}

Bedenklich an Projekten wie dem „Supergrid“ im OffshoreBereich bzw. den Bemühungen der Desertec-Industrial-Initiative ist, dass mit Projekten dieser Größenordnung auch im Bereich regenerativer Energien monopolistische Strukturen geschaffen werden. Nicht zuletzt war die Dezentralisierung der Energieproduktion mittels erneuerbarer Energien lange Zeit ein schlagkräftiges Argument, deren Ausbau voranzutreiben und dadurch die Dominanz der großen Energieversorger zu brechen. Nun besteht die Gefahr, dass die zukünftige Energieversorgung erneut in ein monopolistisches Korsett gezwängt wird und dadurch die Energiepreise weiter hoch gehalten werden. Vor diesem Hintergrund erscheint es angebracht, die Bedeutung des ländlichen Raumes als Standort einer dezentralen Energieversorgung neu zu diskutieren.

Eine mögliche Strategie zur Optimierung der Raumnutzung im ländlichen Raum besteht beispielsweise im Ausbau von regenerativen Kombikraftwerken. Diese können - durch eine intelligente Vernetzung von Standorten mit unterschiedlichen natürlichen Voraussetzungen für erneuerbare Energien - fossile und nukleare Großkraftwerke substituieren. Der Vorteil dieses Ansatzes ist, dass die spezifischen „Talente“ der beteiligten Technologien genutzt bzw. ihre Nachteile ausgeglichen werden. Eine Kombination von Wind-, Solar- und Biomassekraftwerken bietet die Möglichkeit, dass die intermittierenden Energieformen Wind- und 
Solarenergie von der grundlastfähigen Biogastechnologie gestützt werden. Durch diese Abstimmung ließe sich ein unkoordinierter und damit überdimensionierter Ausbau von erneuerbaren Energien vermeiden. Bei zu viel Strom im Netz könnte dieser mittels Pumpspeicherkraftwerken gespeichert und bei steigender Stromnachfrage erneut ins Netz eingespeist werden (Institut für Solare Energieversorgungstechnik 2008: 6 ff.). Der Ausbau von Kombikraftwerken kann jedoch nur erfolgreich sein, wenn es gelingt, den erneuerbaren Energien einen verstärkten Zugriff auf energetisch hochwertige Flächen - auch im ländlichen Raum - zu ermöglichen. Der Raumplanung obliegt es hierbei, die Möglichkeiten und Grenzen von erneuerbaren Energien zu konkretisieren. Sie steht in der Verantwortung, die gesellschaftliche Akzeptanz einer Energiewende dadurch zu erhöhen, dass den erneuerbaren Energien ertragreiche Flächen in Abstimmung mit konkurrierenden Nutzungen zugewiesen und ihr Ausbau auf - aus ökologischer, ökonomischer und sozialer Sicht - bedenklichen Flächen eingeschränkt wird. Des Weiteren gilt es, den Bürgern die Vorteile einer dezentralen Energieversorgung vor Augen zu führen. Die Gelegenheit, beispielsweise Anteile an sogenannten Bürger-Windparks erwerben zu können, wird dabei helfen, die tief verwurzelte Skepsis der Menschen gegenüber regenerativen Technologien abzubauen. Partizipation in Form von Mitspracherecht und Mitgestaltungsprivilegien sowie die Gewährleistung des Zugangs zu billigem und umweltfreundlich produziertem Strom werden damit zu entscheidenden Faktoren eines erfolgreichen Ausbaus von erneuerbaren Energien.

\section{Literatur}

Agentur für Erneuerbare Energien (2009): Erneuerbare Energien 2020. Potenzialatlas Deutschland. Berlin.

Alpha Ventus (2010): Der erste deutsche Offshore-Windpark. Online unter: http://www.alpha-ventus.de (letzter Zugriff am 11.05.2010).

Arbeitsgemeinschaft Energiebilanzen (AGEB) (2009): Energieverbrauch in Deutschland - Daten für das 1.-4. Quartal. http://www. ag-energiebilanzen.de/viewpage.php?idpage $=118$ (letzter Zugriff am 15.04.2010).

ARGE Monitoring PV-Anlagen (2005): Photovoltaik-Freiflächenanlagen - Aktuelle Erfahrungen und Konfliktlinien. Dokumentation eines Workshops vom 21. bis 23.03.2005 im Gustav StresemannInstitut. Bonn.

ARGE Monitoring PV-Anlagen (2007): Leitfaden zur Berücksichtigung von Umweltbelangen bei der Planung von PV-Freiflächenanlagen. Studie im Auftrag des Bundesministeriums für Umwelt, Naturschutz und Reaktorsicherheit. Hannover.

Bard, J. (2006): Windkraft, Wasserkraft und Meeresenergie - Technik mit sozialer, ökologischer und ökonomischer Akzeptanz. Online unter: http://www.fvee.de/fileadmin/publikationen/Themenhefte/ th2006/th2006_02_03.pdf(letzter Zugriff am 06.05.2010).
Bayerisches Staatsministerium des Innern (2009): Freiflächen-Photovoltaikanlagen. Online unter: http://www.innenministerium. bayern.de/imperia/md/content/stmi/bauen/rechtundtechnikundbauplanung/_baurecht/rundschreiben/photovoltaik.pdf (letzter Zugriff am 15.12.2010).

Becker, D. (2010): Photovoltaik geht Offshore. In: Erneuerbare Energien 5, 58-59.

Bosch, S. (2008): Kulturlandschaften und Erneuerbare Energien - Auswirkungen auf Natur und Landschaft: Ökonomische Bedeutung und ökologische Probleme der Biogasgewinnung. In: Verein Rieser Kulturtage e. V. (Hrsg.): Rieser Kulturtage - Eine Landschaft stellt sich vor. Dokumentation Band XVII. Nördlingen, 125-128.

Bosch, S. (2010): Sauberer Strom aus der Wüste - Der Ausbau von konzentrierenden solarthermischen Systemen zur Sicherung der Energie- und Wasserversorgung in der EUMENA-Region. Augsburg. $=$ Geographica Augustana, Bd. 8.

Bosch, S.; Peyke, G. (2008): Energiepflanzenbau und konkurrierende Flächennutzungen - neue Strukturen in der Landwirtschaft und ihre Optimierung mittels GIS. In: Strobl, J.; Blaschke, T.; Griesebner, G. (Hrsg.): Angewandte Geoinformatik 2008 - Beiträge zum 20. AGIT-Symposium. Salzburg, 450-455.

Brücher, W. (2008): Erneuerbare Energien in der globalen Versorgung aus historisch-geographischer Perspektive. In: Geographische Rundschau 60, 1, 4-12.

Bundesamt für Bauwesen und Raumordnung (BBR) (2010): Raumverträgliche Biomasseproduktion - Handlungsmöglichkeiten der Regionalplanung. In: Bundesinstitut für Bau-, Stadt- und Raumforschung (BBSR) (Hrsg.): Informationen aus der Forschung des BBSR, $1,10$.

Bundesamt für Naturschutz (BfN) (Hrsg.) (2010): Natura 2000. Kooperation von Naturschutz und Nutzern. http://www.bfn.de/ fileadmin/MDB/documents/service/BfN_Broschuere_Management_Nutzer.pdf (letzter Zugriff am 13.10.2010).

Bundesamt für Seeschifffahrt und Hydrographie (BSH) (2009): Genehmigung von Offshore-Windenergieparks. Online unter: http:// www.bsh.de/de/Meeresnutzung/Wirtschaft/Windparks/index.jsp (letzter Zugriff am 16.07.2010).

Bundesministerium für Umwelt, Naturschutz und Reaktorsicherheit (BMU) (2007): Entwicklung der Offshore-Windenergienutzung in Deutschland. Berlin.

Bundesministerium für Umwelt, Naturschutz und Reaktorsicherheit (BMU) (2010): Bundestag beschließt Änderung des EEG. Online unter: http://www.erneuerbare-energien.de/inhalt/45993/main (letzter Zugriff am 02.11.2010).

Bundesministerium für Wirtschaft und Technologie (BMWi) (2010): Zulassungsverfahren für die Errichtung von PV-Anlagen in Deutschland. Berlin.

Bundesverband WindEnergie (2010): Windpotenzial Deutschland. Online unter: http://www.wind-energie.de/de/themen/windpotenzial-deutschland/ (letzter Zugriff am 03.05.2010).

Desertec Foundation (2010): Red Paper - Das Desertec Konzept im Überblick. Online unter: http://www.desertec.org/fileadmin/downloads/desertec-foundation_redpaper_3rd-edition_deutsch.pdf (letzter Zugriff am 14.04.2010).

Deutsches Zentrum für Luft- und Raumfahrt (DLR) (2007): AquaCSP - Concentrating Solar Power for Seawater Desalination. Stuttgart.

Dix, A.; Schenk W. (2007): Historische Geographie. In: Gebhardt, H.; Glaser, R.; Radtke, U.; Reuber, P. (Hrsg.): Geographie - Physische Geographie und Humangeographie. Heidelberg, 816-829.

Elsam (2002): Horns Rev Offshore-Windpark. Ein bahnbrechendes Windkraftwerk in der Nordsee. Online unter: http://www.hornsrev.dk/nyheder/brochurer/Horns_Rev_TY.pdf (letzter Zugriff am 09.05.2010)

EuPD Research (2008): The German Photovoltaic Market 2007/2008 - From Sales to Strategic Marketing. Bonn. 
Europäische Union (EU) (2009): Richtlinie 2009/28/EG des Europäischen Parlamentes und des Rates vom 23. April 2009 zur Förderung der Nutzung von Energie aus erneuerbaren Quellen und zur Änderung und anschließenden Aufhebung der Richtlinien 2001/77/EG und 2003/30/EG. Brüssel.

Fachagentur Nachwachsende Rohstoffe (FNR) (2007): Handbuch Bioenergie-Kleinanlagen. Straubing.

Fachverband Biogas (2005): Biogas - das Multitalent für die Energiewende. Freising.

Forsa (Gesellschaft für Sozialforschung und statistische Analysen $\mathrm{mbH}$ ) (2009): Umfrage zum Thema „Erneuerbare Energien“ 2009. Online unter: http://www.unendlich-viel-energie.de/fileadmin/content/Presse/Ergebnisse_forsa09.pdf (letzter Zugriff am 15.06.2010).

Gille, D. (2010): Startschuss: Millionen Watt wandern durch die Nordsee. In: Erneuerbare Energien 6, 24-27.

Institut für Solare Energieversorgungstechnik (ISET) (2008): Das regenerative Kombikraftwerk. Kassel.

juwi solar GmbH (2010): Referenzen. Online unter: http://www.juwi. de/solar/referenzen.html (letzter Zugriff am 25.05.2010).

Kanning, H.; Buhr, N.; Steinkraus, K. (2009): Erneuerbare Energien - Räumliche Dimensionen, neue Akteurslandschaften und planerische (Mit)Gestaltungspotenziale am Beispiel des Biogaspfades. In: Raumforschung und Raumordnung 67, 2, 142-156.

Kratzsch, C. (2009): Offshore-Schmiede Großbritannien. In: Erneuerbare Energien 8, 32-36.

Landesregierung Brandenburg (2009): Windkrafterlass - Gemeinsamer Erlass des Ministeriums für Infrastruktur und Raumordnung und des Ministeriums für Ländliche Entwicklung, Umwelt und Verbraucherschutz (16. Juni 2009). Potsdam.

Lessner, A. (2009): Die Kraft des Meeres. In: Erneuerbare Energien $11,78-80$.

Lessner, A. (2010a): Wie ein Korken im Wasser. Entwicklung von schwimmenden Offshore-Fundamenten steht am Anfang. In: Erneuerbare Energien 2, 18-21.

Lessner, A. (2010b): Widerstand formiert sich. In: Erneuerbare Energien 4, 76-79.

Naturschutzbund Deutschland (NABU) (2010): Zehn-Punkte-Papier Biogas: Grundsätze für eine naturverträgliche Produktion. Online unter: http://niedersachsen.nabu.de/imperia/md/content/niedersachsen/resolutionen/7.pdf (letzter Zugriff am 14.05.2010).

Nohl, W. (2001): Ästhetisches Erlebnis von Windkraftanlagen in der Landschaft. In: Naturschutz und Landschaftsplanung 33, 12, 365-372.
Nohl, W. (2010): Landschaftsästhetische Auswirkungen von Windkraftanlagen. In: Schönere Heimat - Erbe und Auftrag 99, 1, 3-12.

Ott, M. (2006): Top-Silagen für Top-Biogaserträge. In: Mais 2, 48.

Peters, J. (2010): Landschaft als Energieressource - Biomasseproduktion und die Auswirkungen auf die Kulturlandschaft. Beitrag bei der Fachtagung „Raumplanung und die steigende Nutzung von Bioenergie" am 28. September 2010 in Wetzlar. Online unter: http://www.na-hessen.de/downloads/10n102biomassekulturlandschaft.pdf (letzter Zugriff am 13.12.2010).

Peters, J.; Torkler, F.; Hempp, S.; Hauswirth, M. (2009): Ist das Landschaftsbild „,berechenbar“? Entwicklung einer GIS-gestützten Landschaftsbildanalyse für die Region Uckermark-Barnim als Grundlage für die Ausweisung von Windeignungsgebieten. In: Naturschutz und Landschaftsplanung 41, 1, 15-20.

Plank, J. (2004): Biogas: Wärme und Strom vom Acker. Optimale Wärmenutzung ist wichtiges Kriterium bei Planung von modernen und dezentralen Biomasseheizkraftwerken. In: Landwirtschaftliche Mitteilungen 10, 8-9.

Rempel, H. (2008): Globale Verfügbarkeit nicht-erneuerbarer Energierohstoffe. In: Geographische Rundschau 60, 1, 22-31.

Reuning, A. (2010): Untergrundbewegung. In: Erneuerbare Energien 7, 24-26.

Scheidler, A. (2010): Verunstaltung des Landschaftsbildes durch Windkraftanlagen. In: Natur und Recht 32, 525-530.

Schultz, M. (2010): Das Energiekonzept der Bundesregierung. Online unter: http://www.lew-forum-schule.de/cms_ForumSchule_inter/ downloads/Hochschultag_2010_Michael_Schultz.pdf (letzter Zugriff am 30.10.2010).

Sebald, C. (2010): Mehr als ein laues Lüftchen. Der alte Glaube, dass sich Windkraft in Bayern nicht lohne, ist längst widerlegt. In: Süddeutsche Zeitung Nr. 149 vom 02.07.2010, 42.

Tobias, K.; Jung, C.; Fallen, M.; Puga, J.-F. (2003): Hinweise zur Planung von Windenergieanlagen auf Waldstandorten. Kaiserslautern.

Wartner, H. (2010): Solarfelder - Verschandelung oder Gewinn für die Kulturlandschaft. Expertengespräch: Wartner \& Zeitzler Landschaftsarchitekten bdla + Stadtplaner. Online unter: http://www. wartner-zeitzler.de (letzter Zugriff am 06.05.2010).

Weinhold, N. (2009): Der märkische Makel. In: Neue Energie 9, 14-18. 